\title{
High Sensitivity and Full-circle Optical Rotary Sensor for Non-cooperatively Tracing Wrist Tremor with Nanoradian Resolution
}

\author{
Xin Xu, Student Member, IEEE, Zongren Dai, Yifan Wang, Mingfang Li, and Yidong Tan
}

\begin{abstract}
In this paper, a novel optical rotary sensor based on laser self-mixing interferometry is developed for the full-circle rotation measurement. The proposed sensor is convenient to use for it does not need any contact with the target or a cooperative mirror. A prototype is fabricated and tested. The measured results demonstrate a good performance compared with other optical rotary sensors, in terms of the $0.1 \mu \mathrm{rad}$ resolution, the $2.33 \times 10^{-4}$ linearity and $2 \mu \mathrm{rad}$ stability over one hour. Additionally, the repeatability error is below $14.66 \mathrm{mrad}$ under 9-group full-circle tests, which exhibits the potential to be instrumentalized reliably. Error analysis and limitation discussion have been also carried out. Although the accuracy needs further improvement compared with the best rotary sensor, this method has its unique advantages of high resolution, non-cooperative target sensing and electromagnetic immunity. Hence, the proposed optical rotary sensor provides a promising alternative in precise rotation measurement, tremor tracing and nano-motion monitoring.
\end{abstract}

Index Terms-Optical rotary sensor, laser self-mixing interferometry, noncontact measurement.

\section{INTRODUCTION}

$\mathrm{R}$ OTATION measurement plays a crucial role in industrial applications and frontier scientific research, such as automatic control, robotics, precision machining, mask aligner and gravitational wave detection [1]-[5]. Besides the high stability, repeatability, applicability and low complexity, which are important factors for the rotary sensors, the noncontact measurement, full-circle sensing range, high resolution and accuracy are required in the practical applications. Many rotary sensors have been developed in the last several decades based on various working principles, such as the mechanical, electronic and optical methods. Currently, mechanical and electronic sensors are mostly utilized in the rotation measurement. Commercial mechanical turntables and sensors

Manuscript received Month $x x, 2 x x x$; revised Month $x x, x x x x$; accepted Month $\mathrm{x}, \mathrm{xxxx}$. This work was supported by the National Key Research and Development Program of China No. 2020YFC2200204) and the National Science Fund for Distinguished Young Scholars No. 51722506. (Corresponding author: Yidong Tan).

The authors are with the Department of Precision Instruments, Tsinghua University, Beijing 100084, China (email: xx19@mails.tsinghua.edu.cn; $\quad$ dzr18@mails.tsinghua.edu.cn wyf18@mails.tsinghua.edu.cn; $\quad$ limf19@mails.tsinghua.edu.cn are usually limited by the resolution and accuracy. Electromagnetic and capacitive rotary encoders are able to provide accurate angle measurement even in harsh environment [6], [7]. And the resolution can reach micro-radian level and the measuring error is lower than $\pm 24.24 \mu \mathrm{rad}$ over the full-circle range after artificial correction [8], [9]. However, the biggest drawback of the electronic rotary sensors is that the sensing head needs to be fixed on the rotary target. This limitation of non-cooperative target measurement severely narrows the application range of electronic rotary sensors.

As to optical metrology, it has attracted increasing attention due to its nondestructive detection, measuring traceability to the wavelength and strong electromagnetic immunity. Normal interferometric methods need two beams or more to decouple the motions in multi-degree-of-freedom for the angle measurement [10], [11]. Some other optical methods can also measure the angle variation, including auto-collimatic method [12], total internal reflection method [13], grating-based interferometer [14], birefringence heterodyne interferometry [15], surface plasmon resonance [16], self-mixing interferometry [17], and dual-comb interferometry [18]. These methods reach a high resolution, for instance, 4.85 nrad angular displacement has been measured experimentally [10], but the sensing range of the above interferometric methods is strictly limited within several degrees. Circular optical grating encoder is the most precise method of full-circle rotation measurement at present, in which the high precision is owing to the modern grating manufacture [19]. It means that the rotary sensors based on optical grating are expensive and dedicate. More importantly, most optical rotary sensors fail to measure the rotation in the absence of contact or mirror or with non-cooperative target. In order to acquire rotary signals, the sensing part of optical devices are usually attached to the rotary target or an assisted mirror has to be used during the measurement. Only a few ways can achieve noncontact or no-mirror-aid measurement, such as Doppler velocity [20] and digital cameras [21]. Nevertheless, their precision and range are not acceptable. Therefore, efforts should be made to achieve the non-cooperative rotation measurement with high precision, high resolution, full-circle, noncontact and easy-operation at the same time.

In consideration of the requirements above, an optical rotary sensor (ORS) based on laser self-mixing interferometry (LSI) is presented in this paper. Due to the ultra-high sensitivity of LSI 
[22], [23], the method can measure a rotating target without contact or a cooperative mirror. The prototype sensor developed with designed structure is capable of measuring the full-circle rotation with good performance. In condition, the sensor demonstrates the potential in measuring rotation of different materials and monitoring the tremor.

\section{DESIGN AND PRINCIPLE}

\section{A. ORS Structure}

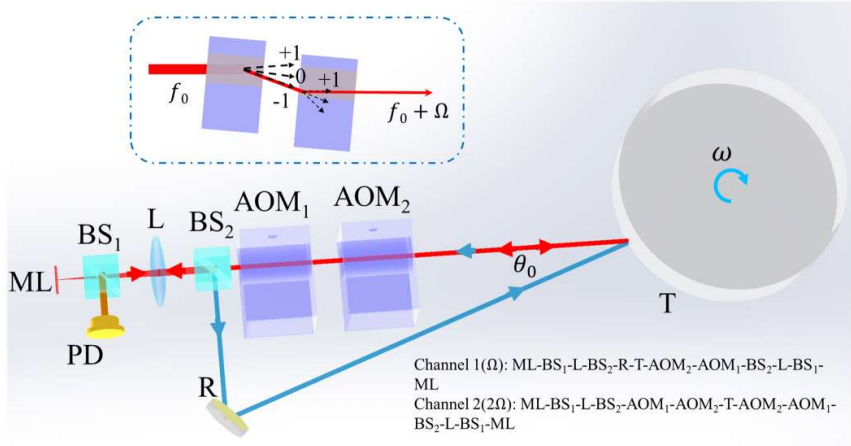

Fig. 1. Schematic diagram of the optical rotary sensor.

ML: microchip laser; BS: beam splitter; L: lens; R: reflector; AOMs: acoustic-optic modulators T: target; PD: photodetector

The optical rotary sensor structure based on laser self-mixing interferometry is designed as shown in Fig. 1. Recently, laser self-mixing interferometry (LSI) has been successfully applied in many fields, including displacement/velocity measurement, laser eavesdropping and biological imaging [22]-[26]. Its high sensitivity and auto-collimation could provide a noncontact measurement of non-cooperative targets [23], which matches well with the demands in rotation measurements.

For the ORS structure, $\omega$ is the angular velocity of the rotary target and $f_{0}$ is the frequency of the output beams. Paths of dual-channel sensing beams have been labeled in Fig. 1. A pair of AOMs provide a heterodyne frequency-shifted modulation of $\Omega$. Sensing beams are incident onto the rotary target, and the reflected or scattered part will feedback into the inner cavity. The feedback signals will cause self-mixing effect on the laser output power, which can be expressed as [23], [27]

$$
\begin{aligned}
& \frac{\Delta I(\Omega)}{I}=\kappa_{1} G(\Omega) \cos \left(2 \pi \Omega t-\phi_{01}+\Delta \phi_{1}\right) \\
& \frac{\Delta I(2 \Omega)}{I}=\kappa_{2} G(2 \Omega) \cos \left(4 \pi \Omega t-\phi_{02}+\Delta \phi_{2}\right)
\end{aligned}
$$

where $\kappa$ is the effective reflection coefficient of the external target, $\phi_{01}$ and $\phi_{02}$ are the initial phase of $\Omega$ and $2 \Omega$ channel, respectively. $G$ is the gain function. $\Delta \phi_{1}$ and $\Delta \phi_{2}$ represent the external phase of the two different channels.

Compared with some other LSI system with more straightforward structure [28]-[30], the proposed method employs a pair of AOMs for the heterodyne modulation. This helps to make the sensor achieve a higher accuracy and resolution, in which the DC drift has been greatly decreased. Furthermore, in microchip lasers, the modulation gain $G$ can reach up to $10^{6}$, which means that the reflectivity of the measured target is as low as $10^{-12}$ when a relative amplitude of a $100 \%$ modulation is achieved [27]. Therefore, it makes the proposed ORS applicable for the rotation measurement without cooperative mirrors.

\section{B. Sensing principle}

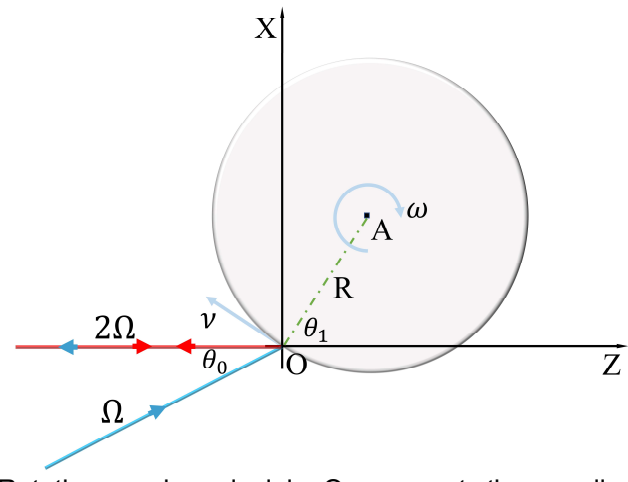

Fig. 2. Rotation sensing principle. O represents the coordinate origin.

Based on the frequency-shifted self-mixing modulation and specific beam-separation structure, we propose a rotation measurement method for column targets. Taking the incident point as the origin $O$ and the channel $2 \Omega$ beam propagation direction as the positive $\mathrm{z}$-axis, a plane rectangular coordinate system is established in Fig. 2. $v$ represents the rotary velocity of the target and $\mathrm{R}$ is the rotary radius. $\theta_{0}$ devotes the angle between two sensing beams and $\theta_{1}$ is the angle between OA and the $\mathrm{z}$-axis, in which the point $\mathrm{A}$ is the center of the rotation target. It is worth to note that we assume the two sensing beams and the rotary plane of the target to be coplanar. The non-coplanar condition will be discussed in the error analysis part.

Clearly, the rotation of the target will lead to the frequency shift for the two sensing beams, namely the Doppler effect. Thereby it will cause the phase variation $\Delta \phi_{1}$ and $\Delta \phi_{2}$ respectively:

$$
\begin{aligned}
& \Delta \phi_{1}=2 \pi \int \Delta f_{1} d t=\frac{2 \pi}{\lambda} \frac{\int\left(\sin \left(\theta_{1}\right)+\sin \left(\theta_{1}-\theta_{0}\right)\right) \omega d t}{R} \\
& \Delta \phi_{2}=2 \pi \int \Delta f_{2} d t=\frac{2 \pi}{\lambda} \frac{\int 2 \sin \left(\theta_{1}\right) \omega d t}{R}
\end{aligned}
$$

where $\lambda$ is the laser wavelength and $c$ is the light velocity in a vacuum. The rotation-path length $S$ is deduced as $S=\int_{0}^{t} v d t=\int_{0}^{t} \omega R d t$ and the relationship between the wavelength and the frequency is $\lambda=\frac{c}{f_{0}}$.Thus, Equation 2 can be deduced as:

$$
\begin{aligned}
& \cos \left(\theta_{1}\right) \cdot \sin \left(\theta_{0}\right) \cdot \frac{2 \pi}{\lambda} \cdot S=\left(1+\cos \left(\theta_{0}\right)\right) \cdot \frac{\Delta \phi_{2}}{2}-\Delta \phi_{1} \\
& \cos \left(\theta_{1}\right) \cdot \frac{2 \pi}{\lambda} \cdot S=\frac{\left(1+\cos \left(\theta_{0}\right)\right) \cdot \Delta \phi_{2}-2 \Delta \phi_{1}}{2 \sin \left(\theta_{0}\right)}
\end{aligned}
$$

Do the square operation of both sides of the Equation 2, and the rotation can be deduced without knowing the incident angle value $\theta_{1}$ as

$$
\theta=\int \omega d t=\frac{\int v d t}{R}=\frac{\lambda}{2 \pi} \frac{\sqrt{\left[\frac{2 \Delta \phi_{1}-\left(1+\cos \left(\theta_{0}\right)\right) \Delta \phi_{2}}{2 \sin \left(\theta_{0}\right)}\right]^{2}+\left[\frac{\Delta \phi_{2}}{2}\right]^{2}}}{R}
$$


$\theta_{0}$ can be calibrated in the experiment as the foreknown parameter [31]. Thereby, the rotation is linked to the changing phases of two incident beams. The proposed method can achieve the noncontact full-circle rotation measurement without assistant mirrors based on its special sensing principle. This feature distinguishes the proposed ORS from other optical methods.

\section{EXPERIMENTAL SETUP AND RESULTS}

\section{A. Prototype}

There are three main parts of the optical rotary sensor, including optical sensing head, signal processing and host computer.

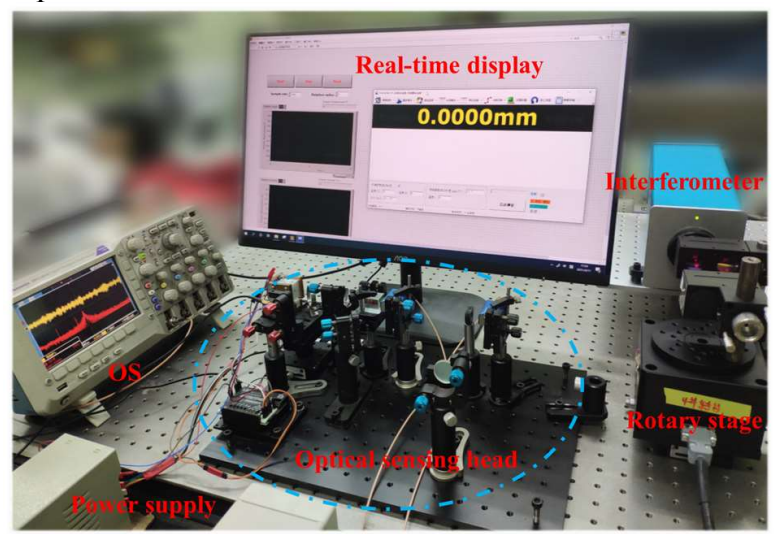

Fig. 3. The optical rotary sensor prototype.

A solid-state microchip $\mathrm{Nd}: \mathrm{YVO}_{4}$ laser is employed for its high gain under frequency-shifted feedback modulation. The laser output beam is linearly polarized and $10.4 \mathrm{~mW}$ of power in a TEM00 mode. The wavelength stability is $2 \times 10^{-8} / \mathrm{h}$ and the power stability is $0.1 \% / 2 \mathrm{~h}$ [32]. Owing to the extremely short photon lifetime compared to the fluorescence lifetime of $\mathrm{Nd}: \mathrm{YVO}_{4}$ crystal, the optical rotary sensor using a solid-state microchip laser has a ultrahigh detecting sensitivity (or signal to noise radio, SNR) [33]. Therefore, the proposed ORS can measure different targets with low reflectivity. This feature will be demonstrated in detail in the discussion part.

The laser output is separated by a beam splitter $\left(\mathrm{BS}_{1}\right.$, 0.96:0.04). A lens $(\mathrm{L}, \mathrm{f}=50 \mathrm{~mm})$ is placed after $\mathrm{BS}_{1}$ for beam collimation. The key designs of the ORS lie in the beam separation and heterodyne modulation, as introduced in the Part 2. The reflective beam of $\mathrm{BS}_{1}$ is detected by a silicon-based photodetector, which contains the modulated signals and the relaxation oscillation [22]. The whole optical sensing head is $300 * 200 * 200 \mathrm{~mm}$, which is smaller than many other optical systems of rotation sensing. It is noted that all the employed optical surfaces have been coated with $1064 \mathrm{~nm}$ anti-reflection films to decrease the effects of parasitic feedback. In the experimental setup, the testing target is an anodized aluminum cylinder, the rotary radius $\mathrm{R}$ is $50 \mathrm{~mm}$ and the angle $\theta_{0}$ is calibrated as $0.8374 \mathrm{rad}[31]$.

Fig. 4 shows the flow of signal processing and sensing display. The obtained signals and the reference signals from the AOM drivers are directly sent to a lock-in amplifier (Zurich Instrument, HF2LI). Phase will be acquired into the host computer by a data acquisition board (NI, USB-6211). Therefore, a real-time rotary sensor is developed containing the angular displacement and velocity with a LabView block diagram.

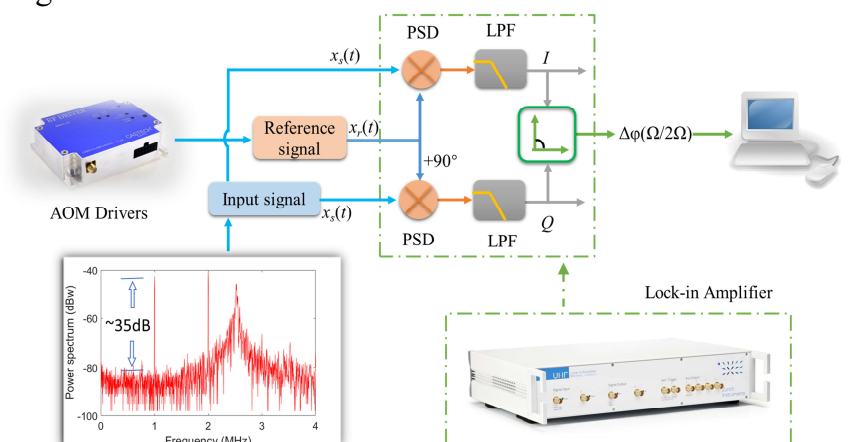

Fig. 4. Dual-channel phase demodulation of the optical rotary sensor. PSD: phase sensitive detector; LPF: low-pass filter.

\section{B. Experimental results}

\section{Stability}

To test the rotation measurement performance, the stability results of the prototype have been firstly obtained. Fig. 5 presents the angular displacement when the rotor keeps stationary. One should note that the results are measured after 1-hour power-on preheating. We first take out a short-term stability test in comparison to the interferometer (LeiCe Technology, LH-3000, resolution $0.1 \mu \mathrm{rad}, 0.485 \mathrm{mrad}$ accuracy over $\pm 0.175 \mathrm{rad}$ ). As Fig. 5(a) shows, the rotation drift is below $80 \mathrm{nrad} / \mathrm{min}$. The theoretical resolution can be calculated from the standard deviation of the stability data [14], which is $16 \mathrm{nrad}$. In this case, nanoradian resolution and ultra-high stability of the rotation sensing can be achieved.

As to long-time stability, we conduct sone-hour stationary angular displacement measurement in four days. The Amplitude Spectrum Density (ASD) results are also given, as illustrated in Fig. 5(c). The results demonstrate that the ORS has a distinguished stability during the short\&long-term test. It is remarkable that the spectrum density of rotation can reach up to $10 \mathrm{nrad} / \mathrm{Hz}^{1 / 2} @ 1 \mathrm{~Hz}$, which is qualified in some precise rotation measurement applications [10].

\section{Rotation resolution}

To determine the resolution of the proposed sensor, step/ square-wave tests through a high-precision rotary stage (PI, Inc. P-562.6CD, 0.1 $\mu \mathrm{rad}$ resolution) have been performed. The corresponding results are shown in Fig. 6. By contrast of the PI, Inc. capacitive sensor, the proposed method proves to be able to distinguish $0.1 \mu \mathrm{rad}$ angular displacement. It is noted that the short-term drift is unavailable but factual, which is caused by and environmental perturbation, including air convection and temperature varying. 

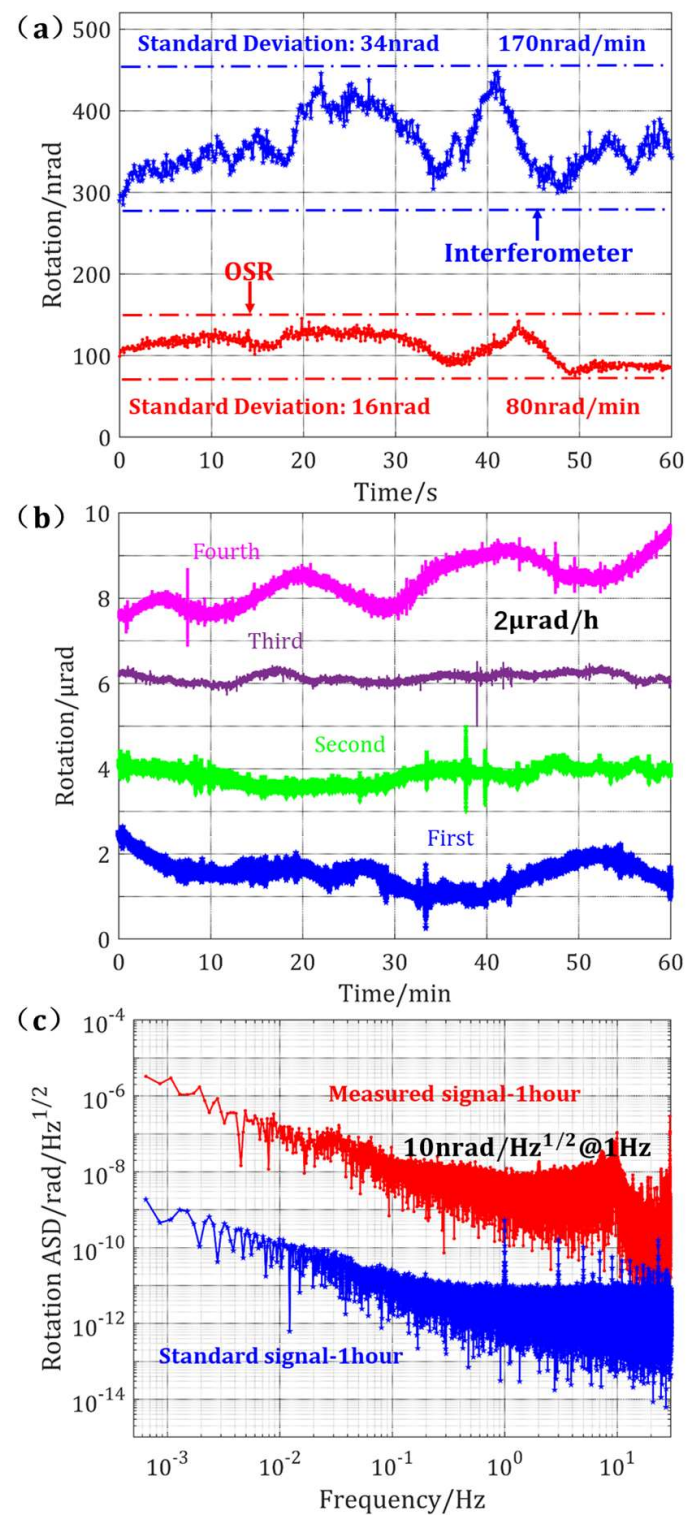

Fig. 5. Stability tests. a. short term (1 min) b. long term (1 hour) c. rotation amplitude spectrum density, optical signal from the actual target \& standard signal from the AOMs driver. Data in $\mathrm{a} / \mathrm{b}$ have been shifted up for convenient display, or the units of the longitudinal axis in $a / b$ are both $100 \mathrm{nrad} / \mathrm{div}$.

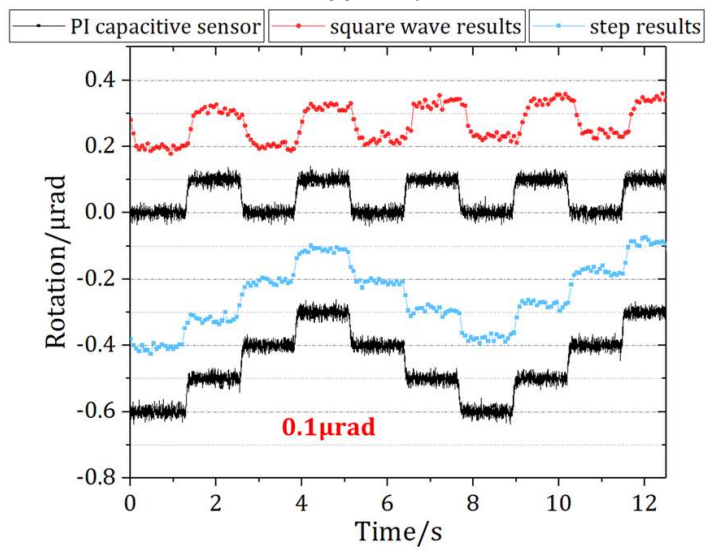

Fig. 6. Resolution tests of square wave and step rotation compared with the capacitive sensor.

\section{Linearity and measuring range}

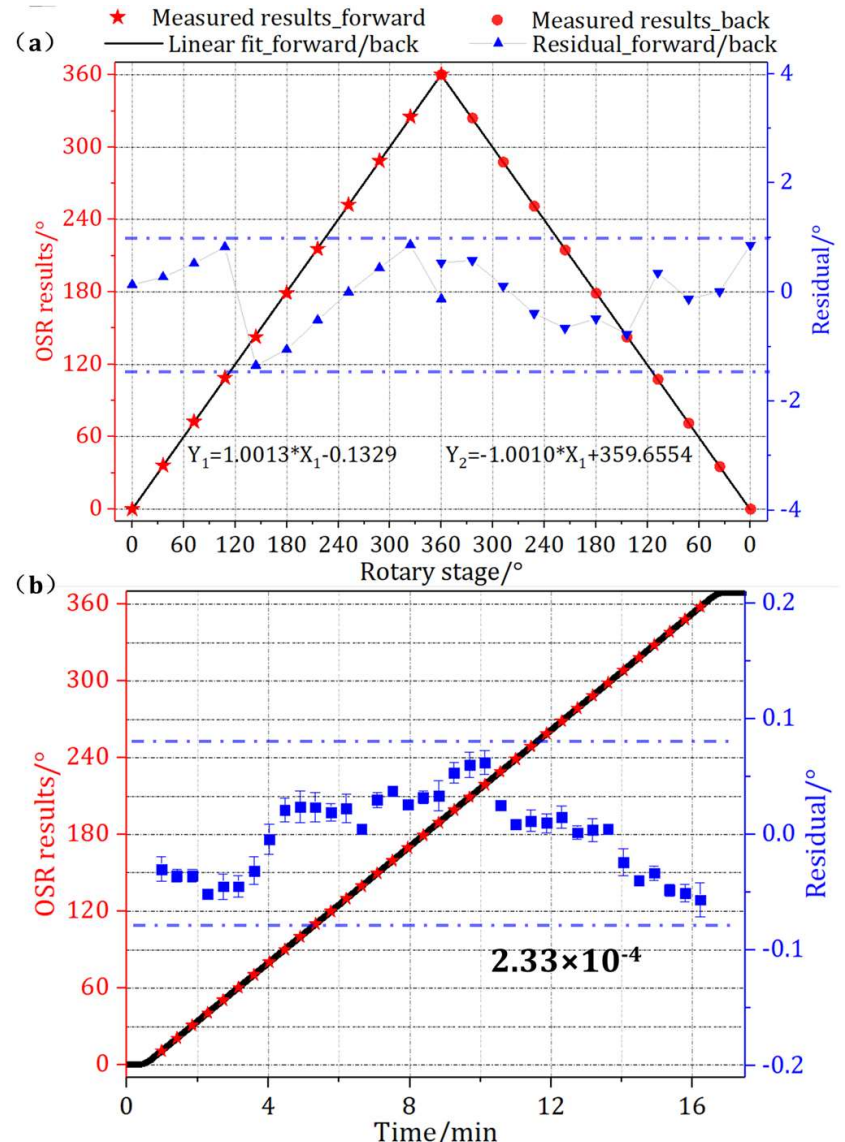

Fig. 7. Linearity test. a. $36^{\circ}$ step of one circle test in the forward and back direction b. four times continuous rotation beyond $360^{\circ}$ range. Stars represent the fitting points of linearity.

Linearity is another important indicator for the rotary sensors. To better test the real linearity, we conduct a $36^{\circ}$ step-by-step rotation of a full-circle and a continuous rotation motion beyond $360^{\circ}$ range with a commercial rotary stage (Beijing Optical Century Instrument, RS211, 10 arcsecond accuracy over $360^{\circ}$ range and 0.33 arcsecond resolution). The nonlinear error in the $36^{\circ}$ step rotation test is assumed to be caused by the start/stop of the rotary stage. The obtained results containing the error bar are presented in Fig. 7(b), showing that the proposed sensor has a linearity of $2.33 \times 10^{-4}$ over $360^{\circ}$ range.

\section{Repeatability test}

Repeatability is defined as the standard deviation of the measured position error between the starting position and the final position [15]. Definitely, a smaller deviation indicates that the rotary sensor has a higher repeatability. To verify the repeatability of the proposed ORS, the rotation stage is operated 9 times forward and backward with a $0.22 \pi$ rad step during the $2 \pi$ rad range. As can been seen in Fig. 8, the experimental results obtained by the proposed sensor and the rotation stage are in good agreement with each other. The standard deviations of 9 groups are lower than $0.84^{\circ}$. The test results clearly demonstrate that the proposed ORS possesses a high measuring repeatability for the rotation sensing. 

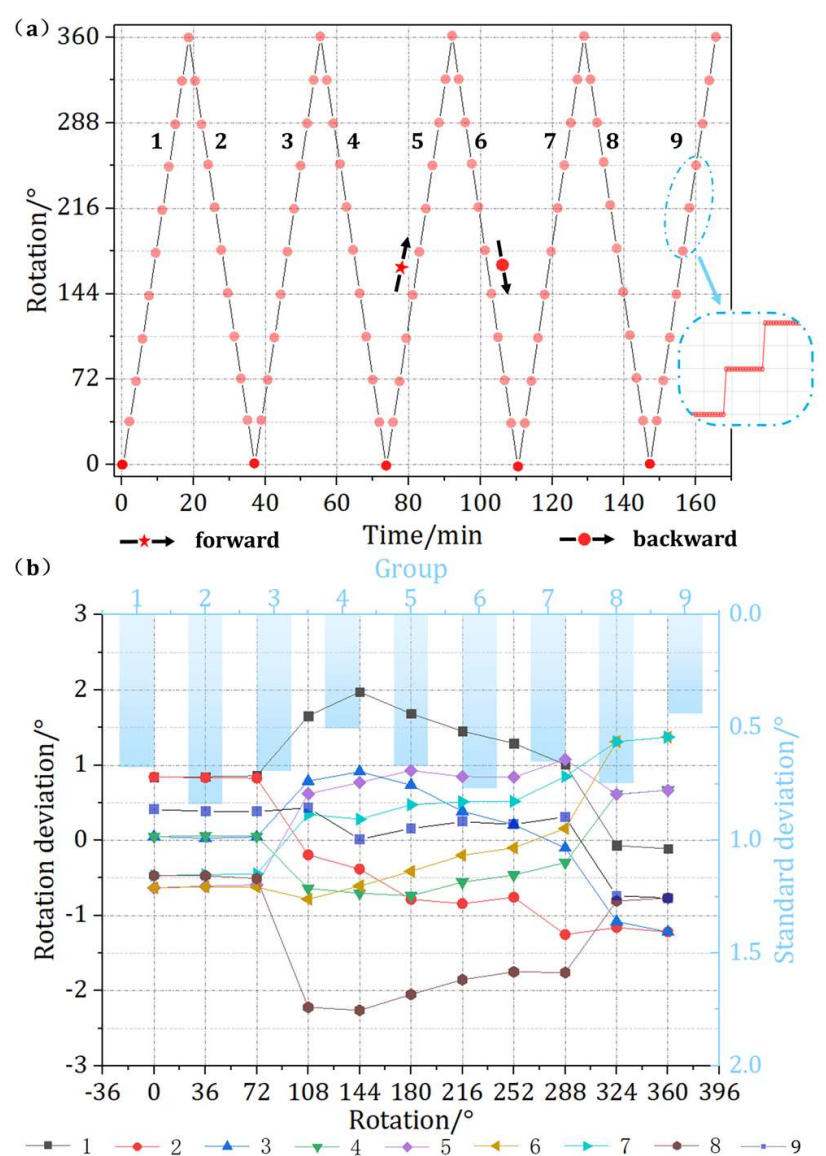

Fig. 8. Repeatability test. a. 9 times test with $0.22 \pi$ rad step of full-circle rotation. b. error results and standard deviation of the repeatability test. The dotted line in Fig. 8(b) represents the difference between measured results and the 9 -group average value, while the column represents the error's standard deviation.

\section{Tremor tracing}

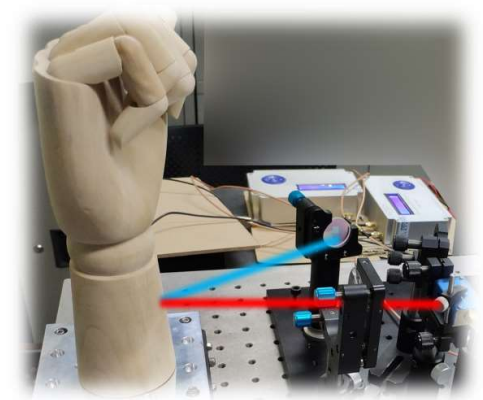

Fig. 9. Experimental setup of tracing the wrist tremor.

Hand/wrist tremor may indicate Parkinsonism in the early stage. The ORS can be employed to trace wrist tremor and recover the rotation information during the tremor process. In the experiment, the rotary stage produces a random rotation motion of a bionic hand, as shown in Fig. 9. Compared with the reference results from the capacitive sensor in the PI, Inc. stage, the ORS can measure the random rotation at the micro-motion level. In this case, the proposed ORS exhibits an excellent traceability for the simulated rotation tremor of the robotic hand, which may provide a precautionary diagnosis method for some tremor diseases. The sensor is also sensitive to the vibration on top of the rotation through the phase measurement. It means that the proposed ORS has the potential to monitor the compound motion after the further study and promotion, in which the vibration tremor and the rotation tremor are both traced.

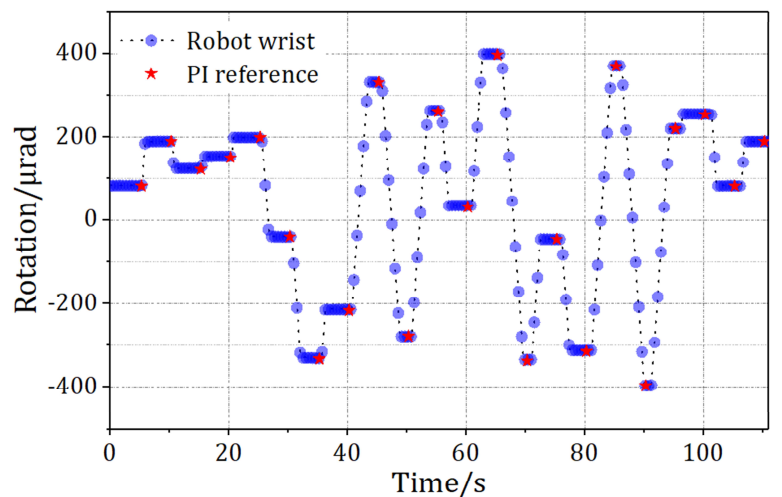

Fig. 10. Recovered results of simulated tremor motions. Five-pointed star gives the reference results from the capacitive sensor in the PI, Inc. stage.

\section{DISCUSSION}

\section{A. Error analysis}

In the prototype, the sensing beams are assumed as parallel to the rotation plane. However, there is a misalignment (incorrect pitch angle) between the rotation plane and two sensing beams. Thus, the final calculation should be modified as follows:

$$
\begin{aligned}
& \theta=F\left(\lambda, R, \beta, \Delta \phi_{1}, \Delta \phi_{2}, \theta_{0}\right) \\
& =\frac{\lambda}{2 \pi} \times \frac{\sqrt{\left[\frac{2 \Delta \phi_{1}-\Delta \phi_{2}\left(1+\cos \left(\theta_{0}\right)\right)}{2 \sin \left(\theta_{0}\right)}\right]^{2}+\left[\frac{\Delta \phi_{2}}{2}\right]^{2}}}{R \cdot \cos (\beta)}
\end{aligned}
$$

where $\beta$ devotes the pitch angle.

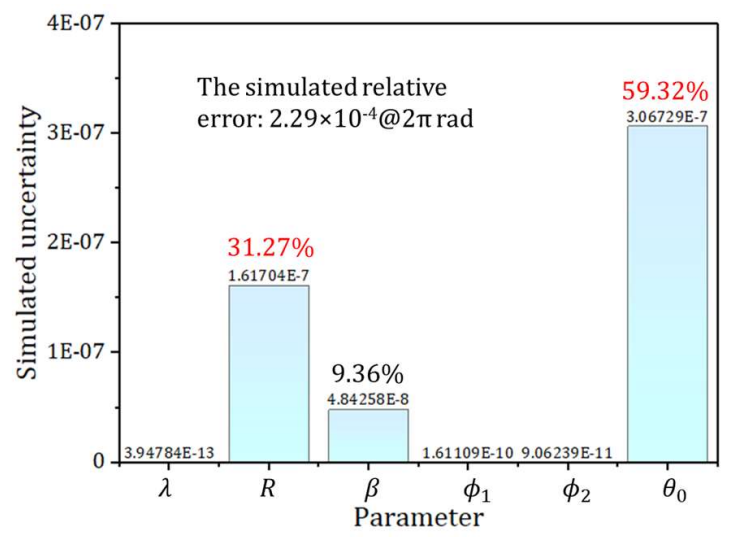

Fig. 11. Uncertainty analysis of each parameter and their proportions to the final relative error.

Based on the designed prototype, we bring out the uncertainty analysis of the rotation measurement. The errors during the full-circle rotation measurement come mainly from the uncertainty of $R$ and $\theta_{0}$. The contribution ratios to the final relative error of the two changing parameters are $31.27 \%$ and $59.32 \%$, respectively. As Fig. 11 shows, the final relative error is calculated to be $2.29 \times 10^{-4}$ over $2 \pi$ rad range when the confidence factor is 2 through the numeral calculation. The analyzed result is in good agreement with the experimental 
results of the linearity. The relative error of the proposed ORS is also better than the measured results of most optical methods from reference [1], [11]-[18], [34], [35]. Accordingly, the proposed ORS is believed to be limited by the uncertainty of $R$ and $\theta_{0}$, which needs further improvement in the future research.

\section{B. Performance analysis}

\section{Testing targets made of different materials}

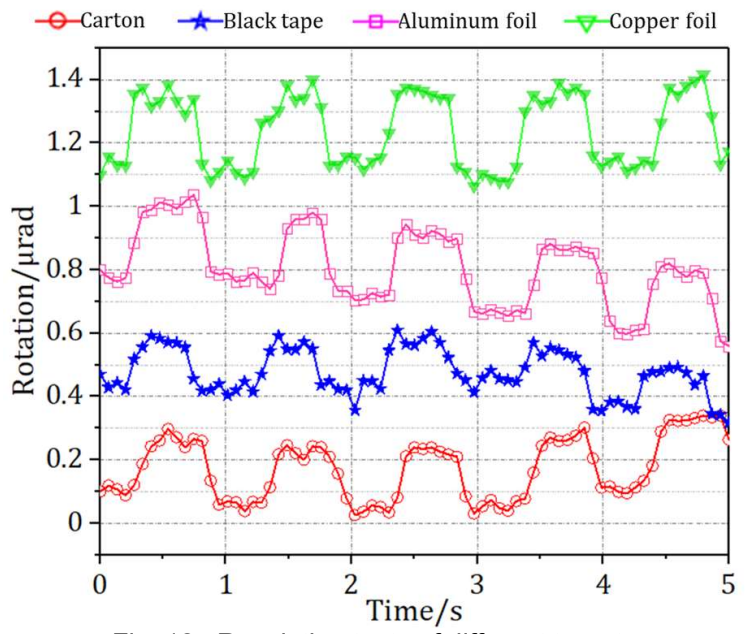

Fig. 12. Resolution tests of different targets.

To further verify the dynamic performance of the proposed sensor, rotation tests with different targets of various materials have been launched out. The obtained results are presented in Fig. 12. It can be seen that the sensor can still distinguish rotation even when the target is of low reflectivity, which verifies the high sensitivity of the proposed ORS based on laser self-mixing interferometry. In addition, all the materials used for tests exhibits a $0.2 \mu \mathrm{rad}$ resolution of angular displacement. Therefore, the proposed ORS has a wide detecting capability and is promising to be used in many applications.

\section{Limitation of the measurement velocity}

Doppler shift caused by the rotation cannot exceed the signal processing bandwidth, thus imposing a limitation on the rotary velocity range. According to the Equation 2, the Doppler shift of the two sensing beams can be deduced as:

$$
\begin{aligned}
\Delta f_{\mathbf{D} 1} & =\left(\sin \left(\theta_{1}\right)+\sin \left(\theta_{1}-\theta_{0}\right)\right) \frac{v}{\lambda} \\
\Delta f_{\mathbf{D} 2} & =2 \sin \left(\theta_{1}\right) \frac{v}{\lambda}
\end{aligned}
$$

When the rotary radium is constant, the highest angular velocity is determined by the sampling bandwidth and the incident angle $\theta_{1}$. To obtain a higher rotary velocity and a better SNR, the incident angle $\theta_{1}$ is set zero in the experiment. The experimental bandwidth is set to be $2 \mathrm{kHz}$, as broader bandwidth means more low frequency noise. In this case, the rotation velocity is allowed to be measured below $1.64 \%$. The measurable angular velocity range can be improved by increasing the demodulation bandwidth to megahertz level with a phasemeter (PT-1313B).

$$
\omega_{\max }=\frac{v_{\max }}{R}=\frac{1}{R} \min \left\{\left|\frac{\Delta f_{\mathbf{D}} \lambda}{\sin \left(\theta_{1}\right)+\sin \left(\theta_{1}-\theta_{0}\right)}\right|,\left|\frac{\Delta f_{\mathbf{D}} \lambda}{2 \sin \left(\theta_{1}\right)}\right|\right\} \approx 1.64^{\circ} / \mathbf{s}
$$

\section{Summary of the proposed ORS and comparison}

To better conclude this method, a performance summary of the proposed ORS is given in Table I. The comparison contains the stability, range, resolution, linearity, repeatability and complexity. The overall comparison has proved that the proposed method has the advantages of high resolution, full-circle range sensing and high accuracy. The comparison with other methods of rotation measurement about the resolution and relative error are also presented in Fig.13. In general, the proposed ORS proves that the advantage over electronic sensors lies in the high resolution of $10^{-8} \mathrm{rad}$, and over other optical sensors lies in the full-circle range and lower relative error. Notably, the ORS enables non-cooperative rotary measurement due to the high sensitivity of laser self-mixing interferometry. This characteristic is promising for many applications, such as satellite posture control, robot gesture recognition and hand tremor monitoring.

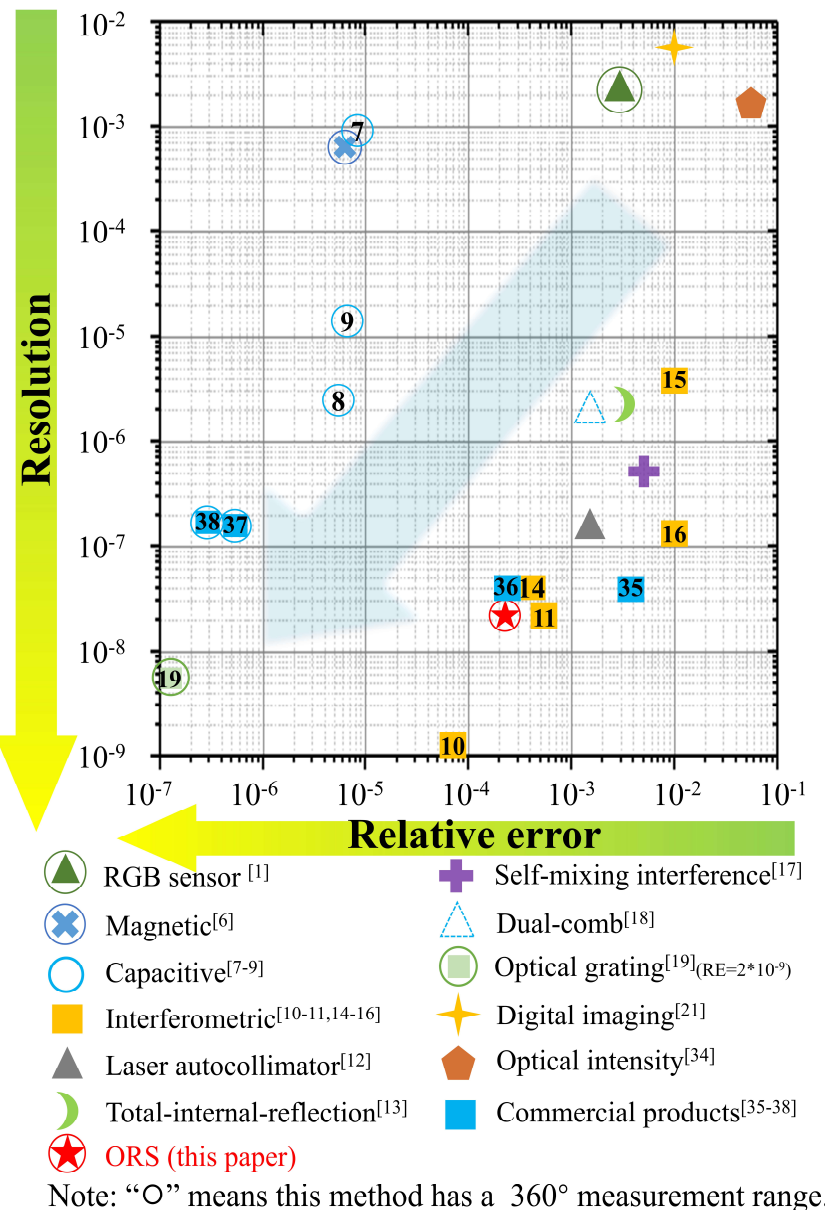

Note: "O" means this method has a $360^{\circ}$ measurement ran

\section{CONCLUSION}

In this paper, a novel optical rotary sensor was developed to measure the full-circle rotation with high resolution and accuracy. Since the high sensitivity of LSI, the sensor is applicable in many rotation measurements due to its noncontact 
sensing feature. The experimental results demonstrated that the rotation measurement with a measured $0.1 \mu \mathrm{rad}$ resolution, $2 \pi$ rad range and $2.33 \times 10^{-4}$ linearity was achieved in the laboratory condition. Theoretical resolution was as low as $16 \mathrm{nrad}$ calculated from the stability data. The precise measurement of the targets made of different materials and the rotation tremor were also achieved. Main errors during the rotation sensing were analyzed to be from the uncertainty of the rotary radius and the angle of two sensing beams. A detailed comparison of the proposed sensor with other optical sensors and commercial angle sensors was presented in Table I and Fig. 13.

Based on the comparison results, the advantages of the optical rotary sensor could be concluded as follows.

1) High resolution and precision: Due to the high sensitivity of laser self-mixing interferometry and precise phase demodulation, the high resolution and precision were obtained compared with normal optical sensors.

2) Noncontact measurement: The sensor did not require contact measurement or any installation of cooperative mirrors.

3) Electromagnetic immunity: Like the other optical sensors, the proposed ORS could work in the electromagnetic environment.

These distinguished characteristics demonstrated that the proposed ORS has promising potential for further applications and instrumentation. In the future, the measurement of compound motion including rotation and displacement should be studied based on the current work. Furthermore, some error compensation algorithms should be applied for improving the accuracy of the ORS.

\begin{tabular}{|c|c|c|c|c|c|c|c|c|}
\hline & This paper & $\begin{array}{c}\text { Laser } \\
\text { interferometr } \\
\mathrm{y}[10]\end{array}$ & $\begin{array}{c}\text { auto-collimat } \\
\text { ic method } \\
{[12]}\end{array}$ & $\begin{array}{l}\text { grating-based } \\
\text { interferomete } \\
\text { ry [14] }\end{array}$ & $\begin{array}{c}\text { Self-mixing } \\
\text { interferometr } \\
y[17]\end{array}$ & $\begin{array}{l}\text { dual-comb } \\
\text { interferometr } \\
\text { y [18] }\end{array}$ & $\begin{array}{c}\text { Optical } \\
\text { intensity [34] }\end{array}$ & $\begin{array}{c}\text { Keysight } \\
5530 \text { Laser } \\
\text { Calibration } \\
\text { System }[35]^{*}\end{array}$ \\
\hline Stability & $\begin{array}{c}80 \mathrm{nrad} \\
@ 1 \mathrm{~min} \\
2 \mu \mathrm{rad} @ 1 \mathrm{~h}\end{array}$ & -- & $\begin{array}{c}2.9 \mu \mathrm{rad} @ \\
110 \mathrm{~s}\end{array}$ & $\underset{\min }{130 \operatorname{nrad} @ 5}$ & $\begin{array}{l}5 \mu \mathrm{rad} \\
\text { (a) } 5 \mathrm{~min}\end{array}$ & -- & $\begin{array}{c}3.5 \operatorname{mrad} @ \\
30 \mathrm{~min}\end{array}$ & $\begin{array}{l}170 \mathrm{nrad} \\
\text { (a) } 1 \mathrm{~min}\end{array}$ \\
\hline Range & $2 \pi \mathrm{rad}$ & $1 \mathrm{mrad}$ & $2.4 \mathrm{mrad}$ & $0.8 \mathrm{mrad}$ & $6.8 \mathrm{mrad}$ & $0.97 \mathrm{mrad}$ & $2 \pi \mathrm{rad}$ & $\pm 0.18 \mathrm{rad}$ \\
\hline Resolution & $0.1 \mu \mathrm{rad}$ & $3 \mathrm{nrad}$ & $0.15 \mu \mathrm{rad}$ & $0.1 \mu \mathrm{rad}$ & $0.48 \mu \mathrm{rad}$ & $1.94 \mu \mathrm{rad}$ & $1.74 \mathrm{mrad}$ & $0.024 \mu \mathrm{rad}$ \\
\hline $\begin{array}{l}\text { Linearity error (\% } \\
\text { of the } \\
\text { range)/Accuracy }\end{array}$ & $0.023 \%$ & $0.006 \%$ & $0.076 \%$ & -- & $0.57 \%$ & $0.21 \%$ & $0.28 \%$ & $0.2 \%$ \\
\hline Repeatability & $\begin{array}{c}14.66 \mathrm{mrad} \\
\text { @ } 2 \pi \mathrm{rad}(9 \\
\text { times })\end{array}$ & -- & -- & $\begin{array}{c}0.1 \mu \mathrm{rad} @ 3 \\
\mu \mathrm{rad}\end{array}$ & -- & -- & $\begin{array}{c}13.1 \mathrm{mrad} \\
\text { @ } 2 \pi \mathrm{rad}(4 \\
\text { times) }\end{array}$ & -- \\
\hline $\begin{array}{l}\text { Easy to } \\
\text { manufacture }\end{array}$ & Moderate & Very complex & Complex & $\begin{array}{c}\text { Very } \\
\text { complex }\end{array}$ & Moderate & Complex & Complex & Moderate \\
\hline
\end{tabular}

*Available in the market

\section{ACKNOWLEDGMENT}

The authors thank Professor Lijiang Zeng, Yan Li and Bin Zhou from Tsinghua University for the help providing the high-precision rotary instruments.

\section{REFERENCES}

[1] Y. Kwon and W. Kim, "Development of a new high-resolution angle sensing mechanism using an RGB sensor," IEEE/ASME Trans. Mechatronics, vol. 19, no. 5, pp. 1707-1715, Oct. 2014.

[2] J. Xue, Z. Qiu, L. Fang, Y. Lu, and W. Hu, "Angular Measurement of High Precision Reducer for Industrial Robot," IEEE Trans. Ins. Mea., vol. 70, pp. 1-10, Oct. 2021.

[3] Glazounov, E. A., Wang, and S., "High-efficiency piezoelectric motor combining continuous rotation with precise control over," Applied Physics Letters, vol. 75, no. 6, pp. 862-865, Aug. 1999.

[4] A. Bag, M. Neugebauer, U. Mick, S. Christiansen, S. A. Schulz, and P. Banzer, "Towards fully integrated photonic displacement sensors," Nature Communications, vol. 11, no. 1, pp. 2915-2921, Jun. 2020.

[5] H. Yan, H. Z. Duan, L. T. Li, Y. R. Liang, and H. C. Yeh, "A dual-heterodyne laser interferometer for simultaneous measurement of linear and angular displacements," Review of Scientific Instruments, vol. 86, no. 12, pp. 123102, Dec. 2015.
[6] Z. Zhang, F. Ni, Y. Dong, C. Guo, M. Jin, and H. Liu, "A novel absolute magnetic rotary sensor," IEEE Trans. Ind. Electron., vol. 62, no. 7, pp. 4408-4419, Jul. 2015.

[7] N. Anandan and B. George, "A wide-range capacitive sensor for linear and angular displacement measurement," IEEE Trans. Ind. Electron., vol. 64, no. 7, pp. 5728-5737, Jul. 2017.

[8] B. Hou, C. Li, Z. Gao, Q. Wei, B. Zhou, and R. Zhang, "Design, optimization, and compensation of a high-precision single-excitation absolute capacitance angular encoder up to \pm 4 ," IEEE Trans. Ind. Electron., vol. 66, no. 10, pp. 8161-8171, Oct. 2019.

[9] H. Wang, K. Peng, X. Liu, Z. Yu, and Z. Chen, "Design and Realization of a Compact High-Precision Capacitive Absolute Angular Position Sensor Based on Time Grating," IEEE Trans. Ind. Electron., vol. 68, no. 4 pp. 3548-3557, Apr. 2021.

[10] H. Yan, Q. Mao, S. Xie, S. Liu, J. Luo, and H.-c. Yeh, "Highly linear sub-nanoradian tilt measurement based on dual-beam interferometry," Optics Letters, vol. 45, no. 10, pp. 2792-2795, May. 2020.

[11] L. Chen, D. Zhang, Y. Zhou, C. Liu, and S. Che, "Design of a high-precision and non-contact dynamic angular displacement measurement with dual-Laser Doppler Vibrometers," Scientific Reports, vol. 8, no. 1, p. 9094, Jun. 2018.

[12] K. Li, C. Kuang, and X. Liu, "Small angular displacement measurement based on an autocollimator and a common-path compensation principle," Review of Scientific Instruments, vol. 84, no. 1, pp. 015108, Jan. 2013.

[13] J.-Y. Lin and Y.-C. Liao, "Small-angle measurement with highly sensitive total-internal-reflection heterodyne interferometer," Applied Optics, vol. 53, no. 9, pp. 1903-1908, Mar. 2014 
[14] H.-L. Hsieh and S.-W. Pan, "Development of a grating-based interferometer for six-degree-of-freedom displacement and angle measurements," Optics Express, vol. 23, no. 3, pp. 2451-2465, Feb. 2015.

[15] H.-L. Hsieh, J.-Y. Lee, L.-Y. Chen, and Y. Yang, "Development of an angular displacement measurement technique through birefringence heterodyne interferometry," Optics Express, vol. 24, no. 7, pp. 6802-6813, Apr. 2016.

[16] S.-F. Wang, M.-H. Chiu, C.-W. Lai, and R.-S. Chang, "High-sensitivity small-angle sensor based on surface plasmon resonance technology and heterodyne interferometry," Applied Optics, vol. 45, no. 26, pp. 6702-6707, Sep. 2006.

[17] S. Zhang, Y.-D. Tan, and S.-L. Zhang, "Non-contact angle measurement based on parallel multiplex laser feedback interferometry," Chin. Phys. B, vol. 23, no. 11, pp. 114202, Nov. 2014

[18] S. Zhou, V. Le, S. Xiong, Y. Yang, K. Ni, Q. Zhou, and G. Wu, "Dual-comb spectroscopy resolved three-degree-of-freedom sensing," Photonics Research, vol. 9, no. 2, pp. 243-251, Feb., 2021.

[19] A. Just, M. Krause, R. Probst, H. Bosse, H. Haunerdinger, C. Spaeth, G. Metz, and W. Israel, "Comparison of angle standards with the aid of a high-resolution angle encoder," Precision Engineering, vol. 33, no. 4, pp. 530-533, Oct. 2009

[20] Y. Zhao, D. Zhu, Y. Chen, Y. Tu, T. Bi, Y. Zhao, B. Yu, and L. Lu, "All-fiber self-mixing laser Doppler velocimetry with much less than 0.1 pW optical feedback based on adjustable gain," Optics Letters, vol. 45, no. 13, pp. 3565-3568, Jul. 2020.

[21] Y.-K. Kim, K.-S. Kim, and S. Kim, "A portable and remote 6-DOF pose sensor system with a long measurement range based on 1-D laser sensors," IEEE Trans. Ind. Electron., vol. 62, no. 9, pp. 5722-5729, Sep. 2015.

[22] K. Otsuka, "Self-mixing thin-slice solid-state laser metrology," Sensors (Basel, Switzerland), vol. 11, no. 2, pp. 2195-2245, Feb. 2011

[23] K. Zhu, C. Hongfang, S. Zhang, Z. Shi, Y. Wang, and Y. Tan, "Frequency-Shifted Optical Feedback Measurement Technologies Using a Solid-State Microchip Laser," Applied Sciences, vol. 9, pp. 109-135, Dec. 2018.

[24] J. Keeley, P. Dean, A. Valavanis, K. Bertling, Y. L. Lim, R. Alhathlool, T Taimre, L. H. Li, D. Indjin, A. D. Rakić, E. H. Linfield, and A. G. Davies, "Three-dimensional terahertz imaging using swept-frequency feedback interferometry with a quantum cascade laser," Optics Letters, vol. 40, no. 6, pp. 994-997, Mar, 2015

[25] A. D. Rakić, T. Taimre, K. Bertling, Y. L. Lim, P. Dean, A. Valavanis, and D. Indjin, "Sensing and imaging using laser feedback interferometry with quantum cascade lasers," Applied Physics Reviews, vol. 6, no. 2, pp Jun, 2019.

[26] T. Taimre, M. Nikolić, K. Bertling, Y. L. Lim, T. Bosch, and A. D. Rakić, "Laser feedback interferometry: a tutorial on the self-mixing effect for coherent sensing," Advances in Optics and Photonics, vol. 7, no. 3, pp. 570-631, Sep, 2015.

[27] E. Lacot, R. Day, and F. Stoeckel, "Coherent laser detection by frequency-shifted optical feedback," Physical Review A, vol. 64, no. 4, pp. 043815 , Sep. 2001.

[28] A. Valavanis, P. Dean, Y. L. Lim, R. Alhathlool, M. Nikolic, R. Kliese, S. P. Khanna, D. Indjin, S. J. Wilson, A. D. Rakic, E. H. Linfield, and G. Davies, "Self-Mixing Interferometry With Terahertz Quantum Cascade Lasers," IEEE Sensors Journal, vol. 13, no. 1, pp. 37-43, Jan. 2013.

[29] L. Scalise, Y. Yu, G. Giuliani, G. Plantier, and T. Bosch, "Self-Mixing Laser Diode Velocimetry: Application to Vibration and Velocity Measurement," IEEE, Trans. Ins. Mea, vol. 53, no. 1, pp. 223-232, Feb. 2015.

[30] J. Perchoux, H. E. Dougan, F. Bony, and A. D. Rakic, "Photodiode-free Doppler velocimeter based on self-mixing effect in commercial VCSELs." IEEE SENSORS 2008 Conference, Oct. 2008

[31] K. Zhu, B. Guo, Y. Lu, S. Zhang, and Y. Tan, "Single-spot two-dimensional displacement measurement based on self-mixing interferometry," Optica, vol. 4, no. 7, pp. 729-735, Jun. 2017.

[32] S. Zhang, S. Zhang, Y. Tan, and L. Sun, "A microchip laser source with stable intensity and frequency used for self-mixing interferometry," Review of Scientific Instruments, vol. 87, no. 5, pp. 113-119, May. 2016

[33] K. Otsuka, "Self-mixing thin-slice solid-state laser Doppler velocimetry with much less than one feedback photon per Doppler cycle," Optics Letters, vol. 40 , no. 20 , Oct. 2015.

[34] S. Das, and B. Chakraborty, "Design and Realization of an Optical Rotary Sensor,” IEEE Sensors Journal, vol. 18, no. 7, pp. 2675-2681, Apr. 2018
[35] Keysight Technologies, "5530 Laser Calibration System,” [Online]. https://www.keysight.com/cn/zh/assets/7018-01910/brochures/5989-935 4.pdf

[36] ArcoBeam, “Collapex STD-3032," [Online] http://www.acrobeam.com/Html/Product/product1/231.html

[37] Precise Instrument, "L-611.9ASD," [Online]. https://www.pi-china.cn/zh_cn/products/rotation-stages/1-611-precisionrotation-stage-1202002/\#specification

[38] Heidenhain, "RON 905," [Online]. ttps://www.heidenhain.com/products/angle-encoders/integral-bearing/ro n-905

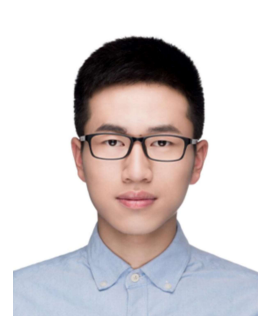

Xin Xu was born in Jiangsu, China, in 1997. He received the B.S. degree in electronic engineering from Nanjing University of Science and Technology, Nanjing, China, in 2019. He is currently working toward the Ph.D. degree in the Department of Precision Instruments, Tsinghua University in Beijing, China. His research interests include precise displacement and distance measurement.

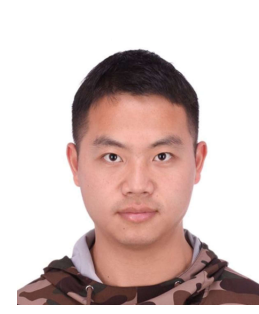

Zongren Dai was born in Sichuan, China, in 1996. He received his bachelor's degree in electronic science and technology from University of Electronic Science and Technology of China, Chengdu, China, in 2018. He is currently working toward the Ph.D. degree in the Department of Precision Instruments, Tsinghua University in Beijing, China.

His current research focuses on biochemical sensing and laser self-mixing interferometry.

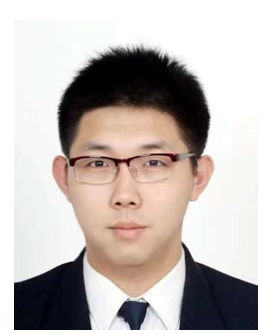

Yifan Wang was born in Hebei, China, in 1996. He received the B.S. degree in Optical Engineering from Tianjin University, Tianjin, China in 2018. He is currently working toward the Ph.D. degree in the Department of Precision Instruments, Tsinghua University in Beijing, China. His current research focuses on laser feedback technique and related applications in fiber laser and microcavity laser.

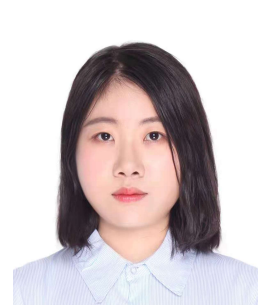

Mingfang Li was born in Hebei, China, in 1997. She received the B.S. degree in college of Information Science and Engineering from the Shandong University, Shandong, China in 2019. She is currently pursuing the Ph.D. degree in the Department of Precision Instrument, Tsinghua University in Beijing, China.

Her research interests include microcavity laser and its applications, laser precision measurement technology and laser feedback technology.

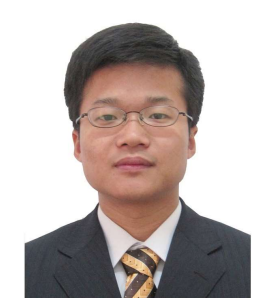

Yidong Tan was born in Jiangsu, China, in 1980 $\mathrm{He}$ received the B.S. degree in 2003 and the Ph.D. degree in 2008 in the Department of Precision Instruments, Tsinghua University in Beijing, China.

He has over 10 years of experience researching photonics. Prof. Tan is currently an Associate Professor in the State Key Lab of Precision Measurement Technology and Instruments, Department of Precision Instruments, Tsinghua University. In 2017, he was with the California Institute of Technology, USA, as a Visiting Scientist. His current research interests include laser interferometer and metrology, laser self-mixing interferometry and biological issue ultrasound imaging.

He was a recipient of the Jin Guofan Young Scholar Fellowship from the China Instrument and Control Society in 2014. He was a recipient of the Newton Advanced Fellowship in 2019. 